\title{
RESPUESTA DEL LITCHI (Litchi chinensis Sonn.) A LA FERTILIZACIÓN CON NPK EN EL NORTE DE OAXACA, MÉXICO
}

\author{
RESPONSE OF LITCHI (Litchi chinensis Sonn.) TO NPK FERTILIZATION IN \\ NORTHERN OAXACA, MÉXICO
}

\author{
Ramiro Maldonado Peralta ${ }^{1}$, Antonio Trinidad Santos ${ }^{1 \star}$, Daniel Téliz Ortíz ${ }^{2}$, \\ Vicente A. Velasco Velasco ${ }^{3}$ y Víctor H. Volke Haller ${ }^{1}$
}

\begin{abstract}
${ }^{1}$ Postgrado en Edafología y ${ }^{2}$ Postgrado en Fitosanidad, Colegio de Postgraduados-Campus Motecillo. Km 36.5 Carretera México-Texcoco.56230, Montecillo, Texcoco, Edo. de México. Fax 01 (595) 95202 00. ${ }^{3}$ Instituto Tecnológico de Valle de Oaxaca, Ex Hacienda de Nazareno. 68120, Xoxocotlán, Oaxaca, Oax. México. Tel y Fax: 01(951) 5170788.
\end{abstract}

*Autor para correspondencia (trinidad@colpos.mx)

\section{RESUMEN}

Se evaluó la respuesta de litchi (Litchi chinensis Sonn.) a la fertilización con NPK en árboles de la var. 'Brewster' de seis años plantados al norte de Oaxaca, en el periodo 2009 - 2011. Se usaron seis proporciones $(0,25,50,75,100$ y $125 \%)$ de la dosis 50-130-50 kg ha-1 de N, $\mathrm{P}_{2} \mathrm{O}_{5}$ y K $\mathrm{O}$ como tratamientos; adicionalmente se probaron otros tres tratamientos: dosis $100 \%+3.6 \mathrm{t} \mathrm{ha}^{-1}$ de composta, dosis $100 \%+18 \mathrm{~kg} \mathrm{ha}^{-1} \mathrm{de}$ elementos menores, y dosis $100 \%+18 \mathrm{~kg} \mathrm{ha}^{-1}$ de elementos menores + $720 \mathrm{~kg} \mathrm{ha}^{-1} \mathrm{de} \mathrm{Ca}(\mathrm{OH})_{2}$. La primera fertilización se aplicó en noviembre de 2009 pero no se encontró respuesta a la fertilización en 2010. En 2010 y 2011 la fertilización se dividió en dos partes. La primera mitad se aplicó el 30 de agosto de 2010, y la otra mitad el 11 de febrero de 2011. Los resultados que aquí se reportan son del periodo 2010 - 2011. Las concentraciones foliares mayores de N, P y K se obtuvieron al inicio de la yema floral con las dosis mas altas de fertilización. La mayor floración y el mayor rendimiento de fruta se lograron con la dosis 100 $\%$ de N-P-K + elementos menores que superó $(\mathrm{P} \leq 0.05)$ al testigo. El mayor contenido de clorofila foliar se obtuvo con el tratamiento $125 \%$, en comparación con el testigo. Los mayores valores de peso de materia seca de brotes vegetativos y de área foliar se lograron con el tratamiento $100 \%+$ composta. El contenido de $\mathrm{N}$ foliar correlacionó con el rendimiento de brotes y con área foliar $(\mathrm{r}=0.72 \mathrm{y} \mathrm{r}=0.65$, respectivamente). Se concluye que los árboles de litchi fertilizados con NPK, composta y elementos menores, aumentaron su concentración foliar de N, P y K, cantidad de flores, rendimiento de fruta, color verde de las hojas, biomasa de nuevos brotes y área foliar. No se encontró respuesta del litchi a la aplicación de hidróxido de calcio.

Palabras clave: Litchi chinensis, área foliar, fertilización, floración, rendimiento, clorofila.

\section{SUMMARY}

Responses of six years old litchi (Litchi chinensis Sonn.) trees cv. 'Brewster' to NPK fertilization at north of Oaxaca state were evaluated from 2009 to 2011. Six dosage treatments $(0,25,5075,100$ and 125 $\%$ ) of the 50-130-50 kg ha-1 of $\mathrm{N}, \mathrm{P}_{2} \mathrm{O}_{5}$ and $\mathrm{K}_{2} \mathrm{O}$ were used. Additional treatments were: $100 \%$ dosage $+3.6 \mathrm{tha}^{-1}$ of compost, $100 \%+18 \mathrm{~kg}$ $\mathrm{ha}^{-1}$ of micronutrients, and $100 \%+18 \mathrm{~kg} \mathrm{ha}^{-1}$ of micronutrients +720 $\mathrm{kg} \mathrm{ha}^{-1}$ of $\mathrm{Ca}(\mathrm{OH})_{2}$. The first fertilization was applied on November 2009, but no response was obtained in 2010. In 2010 and 2011 the fertilization was divided in two parts. The first half was applied in August 30, 2010, and the other in February 11, 2011. The results reported here correspond to the 2010 - 2011 period. Highest concentrations of N, P and $\mathrm{K}$ in the leaves at the flower bud stage were obtained with the highest dosages. The highest amounts of flowers and fruit yield compared to the control were observed with $100 \%$ of NPK + micronutrients. Highest chlorophyll contents were found with the $125 \%$ dosage. The highest values of new shoots dry weight and leaf area were obtained with the $100 \%$ dosage + compost. Positive correlations between foliar nitrogen with new shoots weight and leaf area $(r=0.72$ and $r=0.65$, respectively), were found. It is concluded that litchi trees fertilized with NPK, compost and micronutrients increased N, P and $K$ concentration in the leaves, amount of flowers, fruit yield, green color of leaves, new shoots weight and leaf area. No response to calcium hydroxide application was observed in litchi trees.

Index words: Litchi chinensis, leaf area, fertilization, flowering, yielding chlorophyll.

\section{INTRODUCCIÓN}

El litchi (Litchi chinensis Sonn.), árbol originario de China, es cultivado y apreciado por el sabor agradable de su fruta. Se le conoce desde hace 2000 años a. C., y es la especie más importante de la familia Sapindácea (Menzel, 1984). Fue introducido a México a través del Estado de Sinaloa a principios del siglo XX (Aserca, 2004). Se adapta en las zonas de clima tropical o subtropical cálido, con inviernos secos y veranos cálido-húmedos (Mitra, 2002). Prefiere suelos franco arenosos, con buen drenaje y $\mathrm{pH}$ entre 5 a 8 (Singh y Babita, 2002). La fruta se consume en fresco, deshidratada, enlatada o congelada; se emplea también para la fabricación de licores, así como ingrediente para postres (Cronje et al., 2009).

La producción mundial de litchi es de $2342582.2 \mathrm{t}$, que se cosecha en 755672 ha, con rendimiento medio de $3.1 \mathrm{t}$ ha $^{-1}$ (Menzel y Waite, 2005). Según SIAP (2010), en México se cultivan 4000 ha de litchi distribuidas en 12 estados, con una producción total de 22549 t y un rendimiento promedio de $5.637 \mathrm{t} \mathrm{ha}^{-1}$. En el Estado de Oaxaca se reporta 
un rendimiento promedio de $7.27 \mathrm{t} \mathrm{ha}^{-1}$ (SIAP, 2010). No obstante, en muchos huertos comerciales de los Estados de Oaxaca y Veracruz los rendimientos medios varían entre 1 y 3 t ha $^{-1}$ según observaciones personales realizadas durante los recorridos en la región y la información de los productores.

Durante el ciclo anual de litchi se observan las siguientes fases fenológicas para árboles en producción: 1) Fase de nuevos brotes, que ocurre entre los meses de septiembre a noviembre; 2) Fase de floración, que ocurre de diciembre a febrero según las condiciones de temperatura y humedad; 3) Fase de amarre de fruta, entre febrero y marzo; 4) Fase de desarrollo de fruto, entre marzo y abril; 5) Fase de maduración y cosecha, entre mayo y principios de junio; y 6) Fase de "calmeo" o descanso del árbol, de junio a septiembre (observaciones del autor).

Los rendimientos bajos pueden deberse a varios factores, entre los cuales están el estado nutrimental del árbol, el nivel de fertilidad del suelo, las condiciones climáticas, las horas frío antes de la floración, y el manejo del huerto (Batten y McConchie, 1995; Menzel y Simpson, 1994). Varios huertos de litchi en los Estados de Veracruz y Oaxaca se encuentran en suelos clasificados como Ultisoles, Alfisoles y Oxisoles, que son ácidos y muy pobres en nutrimentos (Soil Survey Staff, 1994).

Nitrógeno es el nutrimento principal para el desarrollo y rendimiento del litchi (Menzel y Waite 2005). La deficiencia de $\mathrm{N}$ afecta el crecimiento de los árboles y las hojas presentan color verde pálido (Menzel, 2002). El N junto con el P, B y Zn limitan la producción de fruta (Mitra, 2002). Uno de los principales problemas de rendimiento es la falta de un programa de nutrición acorde con los requerimientos del cultivo. El uso de equipos que miden clorofila y análisis foliares son importantes para conocer el estado nutrimental de la planta (Menzel et al., 1992; Hieke et al., 2002). Actualmente se cuenta con información de estándares de límites críticos de análisis foliares para el diagnóstico del estado nutrimental de litchi generadas en China, Australia, Sudáfrica, Tailandia y Vietnam (Menzel et al.,1992; Abadia, 1997; Menzel, 2002), que aunque creadas en otros países y en variedades diferentes, en términos generales las concentraciones de nutrimentos son muy semejantes; por tanto, se consideran útiles para el diagnóstico nutrimental en una primera aproximación de los huertos de otras regiones.

El objetivo del presente trabajo fue evaluar el estado nutrimental y la respuesta de litchi de árboles de seis años de la variedad 'Brewster' a la aplicación de NPK, composta, mezcla de elementos menores e hidróxido de calcio, en la región norte del Estado de Oaxaca.

\section{MATERIALES Y MÉTODOS}

El trabajo se llevó a cabo en la localidad de San José Chiltepec, Municipio de Tuxtepec, Oaxaca, en el rancho 'El Refugio'. El sitio se localiza entre las coordenadas $17^{\circ} 57^{\prime}$ 43.6" LN y $96^{\circ} 10^{\prime} 01.6^{\prime \prime} \mathrm{LO}$, en las orillas del río Papaloapan, a $30 \mathrm{msnm}$, con precipitación anual promedio de 3212 $\mathrm{mm}$ y temperatura media anual de $23^{\circ} \mathrm{C}$. El huerto bajo condiciones de temporal (secano) se plantó a $12 \times 12 \mathrm{~m}$ de separación entre árboles, en un Ultisol arcillo-limoso (Soil Survey Staff, 1994) con pH 5.21, materia orgánica $2.3 \%$ (medio), $\mathrm{N} 0.10 \%$ (pobre), $\mathrm{P}_{\text {Bray P-1 }} 12 \mathrm{mg} \mathrm{kg}^{-1} \mathrm{o} \mathrm{ppm}$ (bajo), $\mathrm{K} 0.5 \mathrm{meq} / 100 \mathrm{~g}$ de suelo (bajo), Ca $3.0 \mathrm{meq} / 100 \mathrm{~g}$ de suelo (bajo), $\mathrm{Mg} 0.6 \mathrm{meq} / 100 \mathrm{~g}$ de suelo (muy bajo), Fe $43 \mathrm{ppm}$ (alto), Cu 1.9 ppm (bajo), Zn 1.3 ppm (bajo) y Mn 16 ppm (adecuado).

Antes de la instalación del experimento, en noviembre de 2009 se colectó una muestra compuesta del tejido foliar. Los resultados del análisis nutrimental fueron los siguientes: $\mathrm{N}$ 0.81 , P 0.12, K 0.62, Ca 0.58 y Mg 0.29 expresados en porcentaje, que comparados con los niveles críticos ( $\mathrm{N} 1.50$, $\mathrm{P}$ 0.14, K 0.7, Ca 0.6 y Mg 0.3) son concentraciones bajas (Menzel et al., 1992).

Este trabajo se inició en el ciclo de producción 20092010, y la primera fertilización completa, por tratamientos se aplicó antes de la floración en noviembre de 2009. En este ciclo de producción no se encontró respuesta de litchi a la fertilización.

Los tratamientos de fertilización se generaron de la dosis 50-130-50 $\mathrm{kg} \mathrm{ha}^{-1}$ de N, $\mathrm{P}_{2} \mathrm{O}_{5}$ y $\mathrm{K}_{2} \mathrm{O}$ que equivale a $100 \%$ de fertilización. La dosis $100 \%$ se seleccionó con base en la información disponible sobre fertilización de litchi (Pathak y Mitra, 2010; Kumar, 2010), y en un valor medio de retención de P en el suelo de 30 \% para los Ultisoles y Oxisoles (Soil Survey Staff, 1994). De esta dosis se formaron los tratamientos: $0,25,50,75,100$ y $125 \%$, más tres adicionales: dosis $100 \%$ más 3.6 t ha $^{-1}$ de composta, dosis $100 \%$ más 18 $\mathrm{kg} \mathrm{ha}^{-1}$ de una mezcla comercial de elementos menores, $\mathrm{y}$ dosis $100 \%$ más $18 \mathrm{~kg} \mathrm{ha}^{-1}$ de elementos menores más 720 $\mathrm{kg} \mathrm{ha}^{-1} \mathrm{de} \mathrm{Ca}(\mathrm{OH})_{2}$.

El contenido nutrimental de la compost de caña de azúcar (Saccharum officinarum L.) del Ingenio Tres Valles, Ver., es el siguiente: N, $0.91 \%$; P, $0.8 \%$; K, $0.23 \%$; Ca, $2.16 \%$; $\mathrm{Mg}, 0.27$ \%; Fe, 19986 ppm; Cu, 52 ppm: Zn, 19 ppm; y $\mathrm{Mg}, 845 \mathrm{ppm}$. Estos contenidos son bajos en comparación con estiércoles y composta (Pratt et al., 1973; Castellanos y Reyes, 1982). Se aplicaron $25 \mathrm{~kg}$ de composta a cada árbol para los tratamientos correspondientes, lo que equivale a $3.6 \mathrm{t} \mathrm{ha}^{-1}$. La mezcla comercial de elementos menores de la Casa CARPOL $\mathbb{R}$ que recomienda $18 \mathrm{~kg} \mathrm{ha}^{-1}$ contiene los 
siguientes elementos: $\mathrm{Fe}, 6.0 \%$; $\mathrm{Zn}, 4.0 \%$; $\mathrm{Mn}, 0.7 \%$; $\mathrm{Cu}$, $0.3 \%$; B, $0.05 \%$; Co, $0.003 \%$ y Mo, $0.001 \%$. Se utilizó hidróxido de calcio comercial $\left(\mathrm{Ca}(\mathrm{OH})_{2}\right)$ a la dosis de $25 \mathrm{~kg} /$ árbol, que libera el calcio como un nutrimento y mejora el $\mathrm{pH}$ del suelo.

Como fuente de $\mathrm{N}$ se utilizó fosfonitrito (33-0.03-00); para $\mathrm{P}_{2} \mathrm{O}_{5}$, superfosfato de calcio triple (00-46-00); y para $\mathrm{K}_{2} \mathrm{O}$, cloruro de potasio (00-00-60). La cantidad de cada uno de los fertilizantes químicos por árbol, se calculó con base en una densidad de 144 árboles por hectárea.

En el segundo año de fertilización la dosis de cada uno de los tratamientos se dividió en dos aplicaciones; la primera mitad se hizo el 30 de agosto de 2010 para fortalecer el estado nutrimental del árbol después de la cosecha (Menzel, 1983), y la segunda mitad después de la floración (11 de febrero de 2011). Las cantidades correspondientes de NPK, composta, elementos menores e hidróxido de calcio, se aplicaron uniformemente en toda la superficie alrededor del árbol, desde la base hasta la zona de goteo. Para la distribución de los tratamientos se utilizó un diseño experimental completamente al azar con nueve tratamientos y cuatro repeticiones (Cochran y Cox, 1965), para un total de 36 unidades experimentales.

Las muestras foliares se colectaron a la altura media de la copa, en los cuatro puntos cardinales del árbol (Valdivia et al., 2010). Se hicieron dos muestreos foliares, el primero el 30 de noviembre de 2010 antes de la floración y el segundo el 25 mayo de 2011 después de la cosecha.

Para el muestreo foliar antes de la floración se colectaron cuatro hoja maduras (con 6 a 7 foliolos) del ápice hacia abajo, y después de la cosecha abajo del racimo (Menzel y Waite, 2005). Las hojas se colocaron en hieleras hasta llegar al laboratorio, se lavaron con agua corriente y agua desionizada, y se secaron en una estufa (Thomas Scientific $($ ) a $65^{\circ} \mathrm{C}$, durante $72 \mathrm{~h}$ (Hundal y Arora, 1993). Las hojas secas se molieron en un molino de acero inoxidable Wiley®, con una malla del número 20 , y de esta muestra se tomaron las cantidades necesarias para determinar N, P y K (Chapman y Pratt, 1979). El N se determinó mediante el método semimicro-Kjeldahl (Bremmer, 1965); el P y K mediante digestión húmeda de $0.5 \mathrm{~g}$ de muestra, con una mezcla de ácido $\mathrm{HNO}_{3} / \mathrm{HClO}_{4} 4: 2$. El P se determinó colorimétricamente con el vanadato-molibdato y medido por espectrofotometría, y el K se cuantificó en un fotómetro de emisión de llama por absorción atómica (SpectrAA 220FS®), según Chapman y Pratt (1979).

Se cuantificó el porcentaje de floración de la copa del árbol como variable dependiente. Para ello, el árbol se dividió en cuatro cuadrantes correspondientes a los cuatro pun- tos cardinales que y cada uno representa $25 \%$ del total. En cada cuadrante se evaluó la superficie cubierta de flores y la suma del porcentaje de superficie ocupada por flores en cada cuadrante, representó el porcentaje total de $100 \%$.

El rendimiento de fruto se cuantificó en kilogramos por árbol para cada uno de los tratamientos. Para ello se utilizó una balanza digital (EK3052 $\left.{ }^{\circledR}\right)$ con aproximación de 1 g.

Las mediciones del contenido foliar de clorofila se hicieron con aparato Minolta SPAD-502®) (Soil Plant Analysis Development, Spectrum Technologies Inc. USA.) en el limbo de un foliolo maduro de la hoja, repetidos cuatro veces en los cuatro puntos cardinales de cada árbol; el promedio de estas lecturas se utilizó como variable dependiente de los diferentes tratamientos. Estas mediciones se llevaron a cabo en el mes de noviembre de 2010.

Se evaluaron brotes vegetativos del año en el mes de junio de 2010 en cada uno de los árboles. Para ello se cortaron los brotes vegetativos en $1 \mathrm{~m}^{2}$ de la parte media de la copa del árbol y se pesaron en una balanza granataria (KW-2610 $($ ); se tomaron $100 \mathrm{~g}$ en bolsas de papel para secarlos en estufa (Thomas Scientific ${ }^{\circledR}$ ) a $65^{\circ} \mathrm{C}$ durante $72 \mathrm{~h}$. Se calculó el porcentaje de materia seca y con ello se determinó el peso total de $1 \mathrm{~m}^{2}$ de la superficie del árbol. Posteriormente se multiplicó el peso de materia seca de $1 \mathrm{~m}^{2}$ por la superficie total del árbol. La superficie de la copa del árbol $(A l)$ se calculó con la fórmula de Caballero et al. (1993): $\mathrm{Al}=\pi \mathrm{rg}$, en donde $r=$ radio de la copa, y $g=$ generatriz, que se calcula con $r$.

El área foliar se cuantificó en una superficie de 25 x $25 \mathrm{~cm}$ que representa la 16ava parte de $1 \mathrm{~m}^{2}$. Se pesó el follaje de $0.0625 \mathrm{~m}^{2}$ en los cuatro puntos cardinales de la copa media del árbol. Las referencias indican que hay una buena relación entre el peso de hojas y el área foliar (Garcés y Forcelini, 2011; Barrantes y Gracia, 1989). Para facilitar el trabajo se tomaron de tres hojas maduras con 20 foliolos de cada uno de los cuadrantes, los cuales se pesaron y midieron su área foliar en un medidor LI-3 000 (LICOR, Inc.; Lincoln, NE, USA), que permitió la determinación del área foliar en los $0.0625 \mathrm{~m}^{2}$ por la relación entre el peso y el área foliar. Se sumaron los valores anteriores para obtener el área foliar en $0.250 \mathrm{~m}^{2}$, que es el resultado de $0.0625 \mathrm{~m}^{2}$ por cuatro puntos cardinales muestreados en el árbol. Finalmente, con los valores anteriores se calculó el área foliar total por árbol con base en su superficie total. Se utilizó la misma superficie total por árbol calculada para el peso de brotes vegetativos.

Los datos obtenidos de las variables se procesaron mediante análisis de varianza, comparación de medias con la prueba de Tukey $(\mathrm{P} \leq 0.05)$ y correlaciones entre algunas variables, con el paquete estadístico SAS versión 9.0 (SAS Institute, 2003). 


\section{RESULTADOS Y DISCUSIÓN}

En el Cuadro 1 se presenta el efecto de los tratamientos de fertilización en algunas variables de respuesta del litchi var. 'Brewster' de seis a ocho años de edad en Chiltepec, Tuxtepec, Oaxaca.

\section{Contenidos de N, P y K}

En las Figuras 1, 2 y 3 se presentan las concentraciones de N, P y K en hojas de litchi, respectivamente. Las de N, P y $\mathrm{K}$ fueron mayores antes que después de la floración, con excepción de los tratamientos 0,25 y $50 \%$ de la dosis de referencia de NPK para el N, pero menores después de la cosecha. Esto indica que las hojas acumularon nutrimentos hasta antes de la floración, y después los traslocaron hacia los órganos de producción, como señalaron Fan et al. (2005). También se detectaron incrementos significativos $(\mathrm{P} \leq 0.05)$ de N, P y K foliar al aumentar la dosis de fertilización, lo que evidencia que la planta respondió a la aplicación de NPK en 2009 y 2010. Sin embargo, la concentración foliar de $\mathrm{N}$ se ubicó por abajo del límite crítico inferior, con excepción del tratamiento con la dosis más alta de NPK.

Antes de la floración, con la dosis $125 \%$ de NPK se encontró que la concentración de N (1.50\%) en la hoja se incrementó en $39 \%$ con respecto al testigo (1.08 \%) (Figura 1). El nitrógeno es el nutrimento más importante para el crecimiento y producción de litchi (Menzel y Waite, 2005); $\mathrm{Al}$ respecto, Menzel et al. (1995) y Thomas et al. (1995) demostraron que las deficiencias de $\mathrm{N}$ afectan el crecimiento de brotes, el tamaño y color verde de la hoja, el porcentaje de floración, y el cuajado de fruto y su peso.
La concentración de P foliar (0.22\%) con la dosis 100 $\%$ de NPK más composta fue superior en $69 \%$ que la del testigo $(0.13 \%)$. El abatimiento de la concentración de $\mathrm{P}$ entre floración y cosecha indica que hubo traslocación de este nutrimento de las hojas a los órganos de producción (Figura 2). Según Menzel y Waite (2005), la importancia de $P$ en litchi se refleja en un mayor porcentaje de floración y de fructificación.

La concentración de $\mathrm{K}$ foliar antes de la floración fue de $1.16 \%$ en el tratamiento con $100 \%$ de NPK más elementos menores (Figura 3), y después de la cosecha bajó a 0.87 $\%$; esta diferencia se atribuye a la traslocación de K (Fan et al., 2005). El hecho de que este tratamiento haya producido el mayor rendimiento de fruta, evidencia la importancia del $\mathrm{K}$ en la fructificación, presumiblemente a través de su papel en Ia división celular y el potencial osmótico de la célula como indicaron Lynch et al. (1974). Menzel y Simpson (1987) encontraron que las deficiencias foliares de N, P y K disminuyen el cuajado del fruto de litchi, así como su desarrollo y rendimiento.

\section{Floración, rendimiento, clorofila, peso de brotes $\mathrm{y}$ área foliar}

Hubo diferencias $(\mathrm{P} \leq 0.05)$ entre tratamientos en cuanto a floración, rendimiento de fruto, contenido de clorofila, peso de brotes y área foliar (Cuadro 1), cuyos valores se incrementaron con la fertilización de NPK, como también reportaron Lynch et al. (1974), Menzel y Simpson (1987) y Ray y Singh (1989).

Cuadro 1. Efecto de los tratamientos de fertilización en árboles de litchi variedad 'Brewster' de seis a ocho años de edad, en Tuxtepec, Oaxaca.

\begin{tabular}{|c|c|c|c|c|c|}
\hline Tratamiento & $\begin{array}{l}\text { Floración en } \\
\text { copa }(\%)\end{array}$ & $\begin{array}{l}\text { Rendimiento } \\
\text { (kg/árbol) }\end{array}$ & $\begin{array}{c}\text { Cont. clorofila } \\
\text { (lecturas SPAD) }\end{array}$ & $\begin{array}{l}\text { Peso de brotes } \\
\text { (kg/árbol) }\end{array}$ & $\begin{array}{l}\text { Área foliar } \\
\left(\mathrm{m}^{2} / \text { árbol }\right)\end{array}$ \\
\hline $0 \%$ & $6.75 \mathrm{~d}$ & $0.26 \mathrm{~b}$ & $32.96 \mathrm{~d}$ & $1.38 \mathrm{~d}$ & $151.69 \mathrm{f}$ \\
\hline $25 \%$ & $20.25 \mathrm{bcd}$ & $0.46 \mathrm{~b}$ & $33.76 \mathrm{~d}$ & $3.08 \mathrm{~cd}$ & $170.99 \mathrm{e}$ \\
\hline $50 \%$ & $36.25 \mathrm{abc}$ & $1.55 \mathrm{~b}$ & $38.46 \mathrm{c}$ & $4.37 \mathrm{bc}$ & $187.71 \mathrm{~d}$ \\
\hline $75 \%$ & $28.75 \mathrm{bcd}$ & $1.35 \mathrm{~b}$ & $38.94 \mathrm{c}$ & $6.85 \mathrm{~b}$ & 209.18 c \\
\hline $100 \%$ & $38.75 \mathrm{ab}$ & $1.50 \mathrm{~b}$ & $40.11 b c$ & $13.36 \mathrm{a}$ & $235.81 \mathrm{~b}$ \\
\hline $125 \%$ & $27.00 \mathrm{~cd}$ & $1.88 \mathrm{ab}$ & $45.38 \mathrm{a}$ & $14.02 \mathrm{a}$ & $243.58 \mathrm{~b}$ \\
\hline $100 \%+C$ & $8.50 \mathrm{~cd}$ & $0.30 \mathrm{~b}$ & $40.07 \mathrm{bc}$ & $15.12 \mathrm{a}$ & $274.19 \mathrm{a}$ \\
\hline $100 \%$ + EM & $63.75 \mathrm{a}$ & $3.61 \mathrm{a}$ & $40.62 \mathrm{bc}$ & $12.98 \mathrm{a}$ & $262.52 \mathrm{a}$ \\
\hline $100 \%+\mathrm{EM}+\mathrm{Cal}$ & $17.75 \mathrm{bcd}$ & $0.29 \mathrm{~b}$ & $42.47 \mathrm{~b}$ & $14.66 \mathrm{a}$ & $268.32 \mathrm{a}$ \\
\hline CV (\%) & 11.50 & 20.12 & 2.95 & 11.86 & 2.36 \\
\hline
\end{tabular}




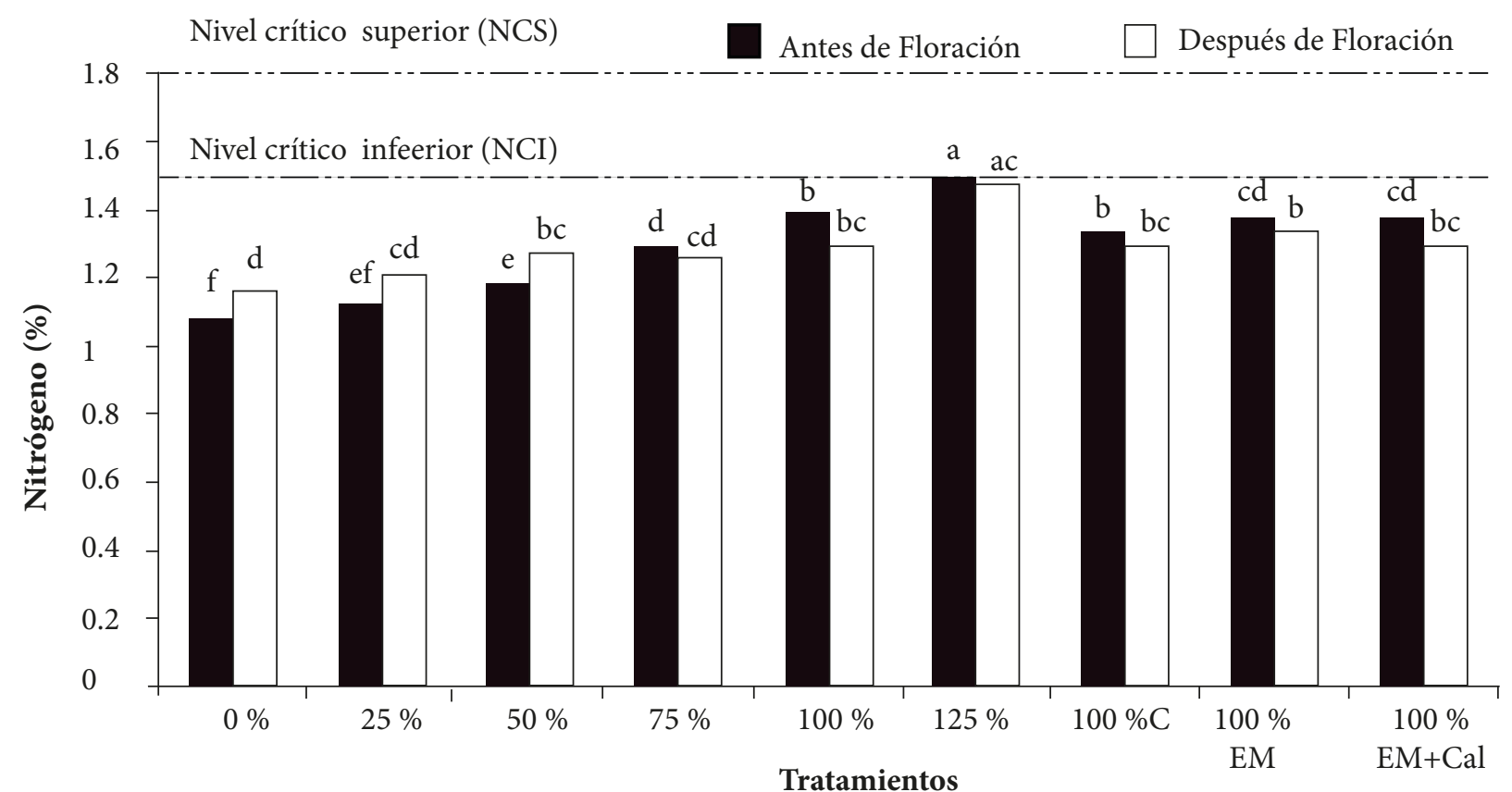

Figura 1.Concentración de $\mathrm{N}$ en hojas de litchi var. 'Brewster' de seis a ocho años de edad, en Tuxtepec, Oaxaca. $\mathrm{C}=$ composta; $\mathrm{EM}=$ elementos menores; $\mathrm{Cal}=\mathrm{Ca}(\mathrm{OH})_{2}{ }^{\circ}$

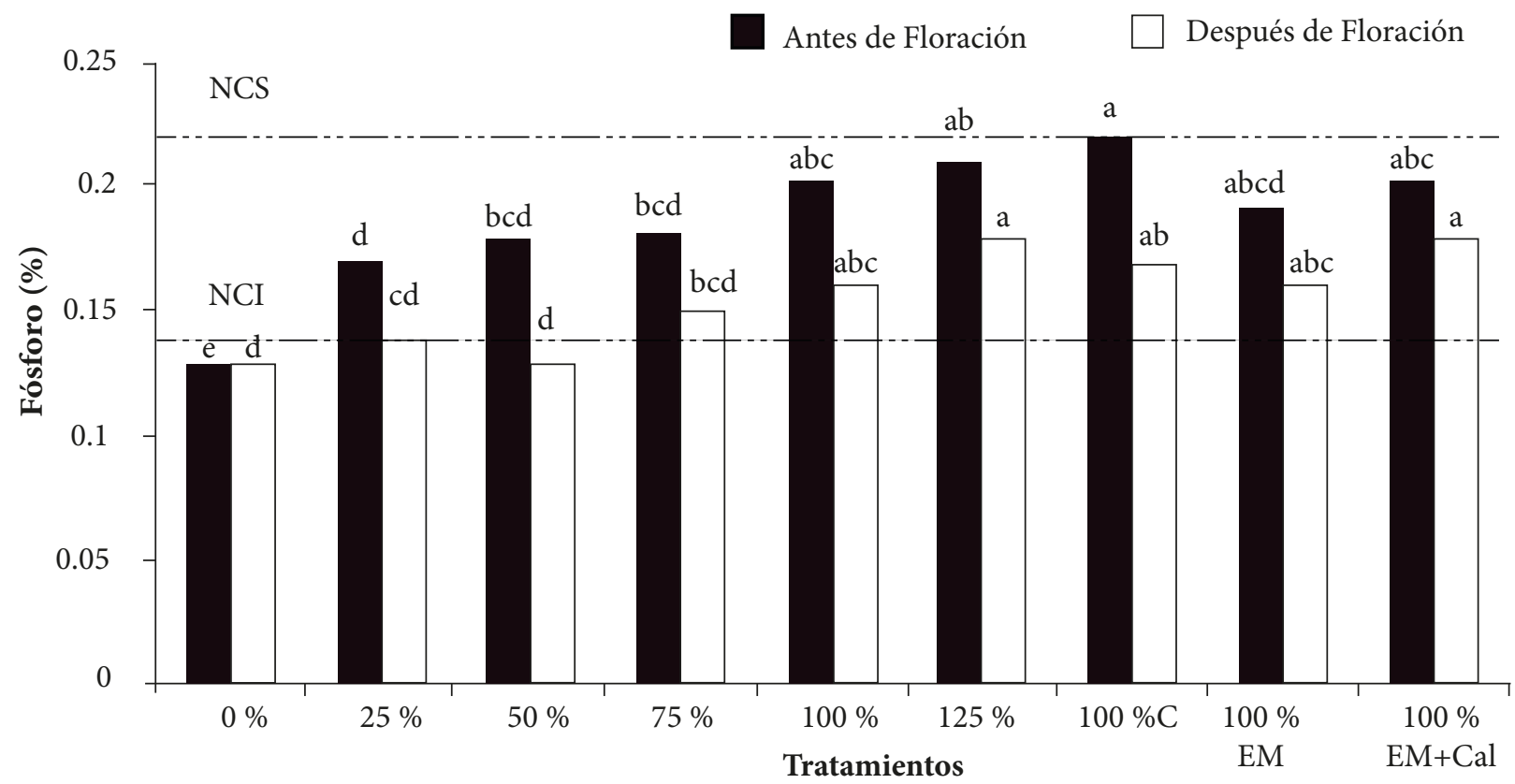

Figura 2. Concentración de $\mathrm{P}$ en hojas de litchi de la var. 'Brewster' de seis a ocho años de edad, en Tuxtepec, Oaxaca. $\mathrm{C}=$ composta; $\mathrm{EM}=$ elementos menores; $\mathrm{Cal}=\mathrm{Ca}(\mathrm{OH})_{2}$.

Los mayores valores de porcentaje de floración (63.7 $\%)$ y de rendimiento de fruto (3.61 kg/árbol) se obtuvieron, como ya se indicó, con la dosis $100 \%$ de NPK más elementos menores, los cuales fueron superiores $(\mathrm{P} \leq 0.05)$ al testigo (6.75 \% y $0.26 \mathrm{~kg} /$ árbol, respectivamente). Estos resultados evidencian la existencia de alguna deficiencia micronutrimental en los árboles estudiados de litchi. 


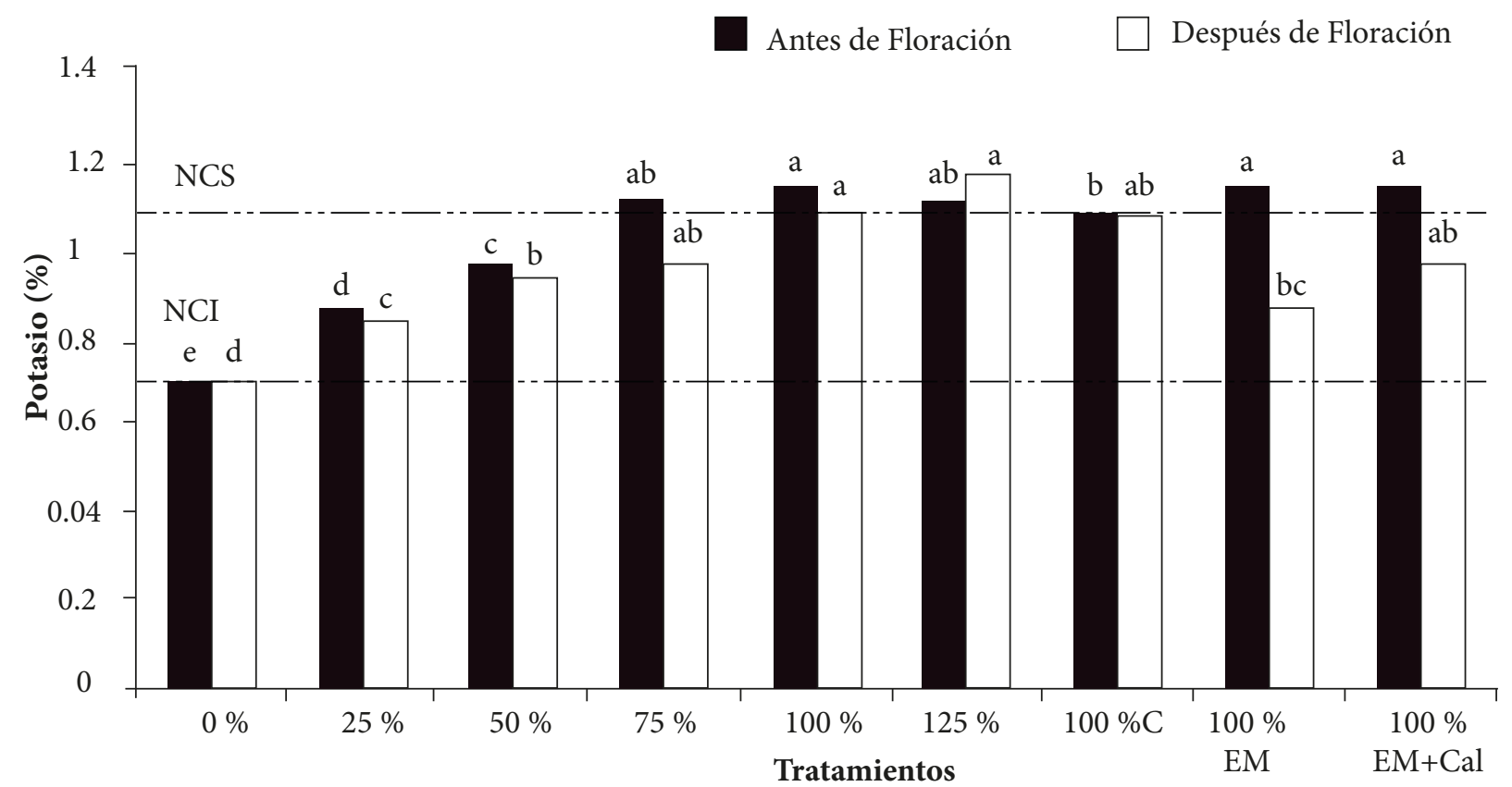

Figura 3. Concentración de $\mathrm{K}$ en hojas de litchi de la var. 'Brewster' de seis a ocho años de edad, en Tuxtepec, Oaxaca. $\mathrm{C}=$ composta; $\mathrm{EM}=$ elementos menores; $\mathrm{Cal}=\mathrm{Ca}(\mathrm{OH})_{2}$.

El color verde de las hojas se determinó con el SPAD que mide el contenido de clorofila, y la mayor lectura se obtuvo con la dosis $125 \%$ de NPK que supera en $38 \%$ a la del testigo. Las lecturas SPAD miden indirectamente la actividad fotosintética de la planta y están relacionadas directamente con la disponibilidad de $\mathrm{N}$ y el contenido foliar de este elemento. Numerosas investigaciones han utilizado esta metodología para evaluar el estado nutrimental de la planta en relación con la disponibilidad de $\mathrm{N}$ en el medio de crecimiento (Heike et al., 2002).

El peso de materia seca de brotes vegetativos del año indica la capacidad de crecimiento de la planta. Los valores más altos (15.12 kg/árbol) se obtuvieron con las dosis 100 $\%$ de NPK más composta, que superaron $(\mathrm{P} \leq 0.05)$ al testigo (1.38 kg/árbol). En general, se observó que el peso de materia seca de los brotes vegetativos se incrementó con la aplicación de cantidades crecientes de NPK, resultados que coinciden con los datos reportados por Menzel y Waite (2005).

La capacidad fotosintética de una planta está directamente relacionada con la superficie foliar expresada como índice de área foliar (Ray y Singh, 1989; Kozlowski et al., 1991). El área foliar de litchi fue mayor $(\mathrm{P} \leq 0.05)$ en los tratamientos que recibieron la dosis $100 \%$ de NPK más composta, $100 \%$ de NPK más elementos menores y $100 \%$ de NPK más elementos menores y cal (274, 262 y $268 \mathrm{~m}^{2}$ por arbol, respectivamente), con respecto al resto de tratamientos. El tratamiento testigo (sin fertilización) presentó menor área foliar (151 $\mathrm{m}^{2}$ por árbol), atribuible a su pobre condición nutrimental, como se muestra en el Cuadro 1. Estos datos evidencian que la fertilización juega un papel importante en el desarrollo de la planta; según Li et al. (2011), en pastos la fertilización nitrogenada duplica la tasa foliar, mientras que la fertilización con P tuvo poco efecto en el área foliar.

\section{Nitrógeno foliar y el peso de brotes y área foliar}

La mayoría de las plantas responden rápidamente a la fertilización nitrogenada en la acumulación de biomasa, mientras que la fertilización fosfatada y potásica promueve la floración y fructificación (Menzel y Waite, 2005).

En la Figura 4 se muestra la relación cuadrática que hay entre la concentración de $\mathrm{N}$ y el rendimiento de brotes nuevos. Dicha relación indica que por cada décimo de porcentaje de incremento de $\mathrm{N}$ en la hoja hay un incremento de $4.55 \mathrm{~kg} /$ árbol de peso de materia seca.

Los resultados de área foliar de litchi var. 'Brewster' (Figura 5) también muestran que esta variable se incrementa al aumentar la concentración de $\mathrm{N}$ en el tejido foliar. De acuerdo con la ecuación lineal, por cada décimo porcentual de incremento de concentración de $\mathrm{N}$ en la hoja hay un incremento en área foliar de $48 \mathrm{~m}^{2}$ por árbol. La información anterior señala que los árboles con mayor área foliar están recibiendo mayor abastecimiento 


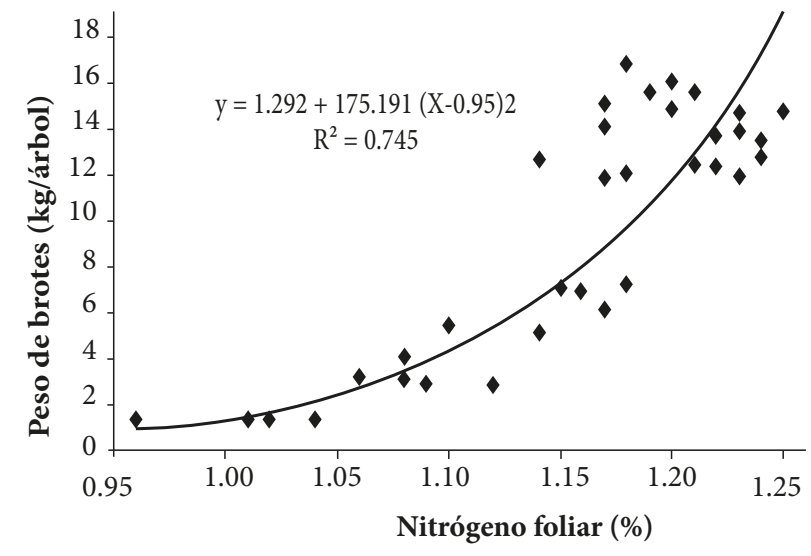

Figura 4. Regresión entre el peso de brotes de árboles de litchi var. 'Brewster' y la concentración de $\mathrm{N}$ foliar, en Tuxtepec, Oaxaca.

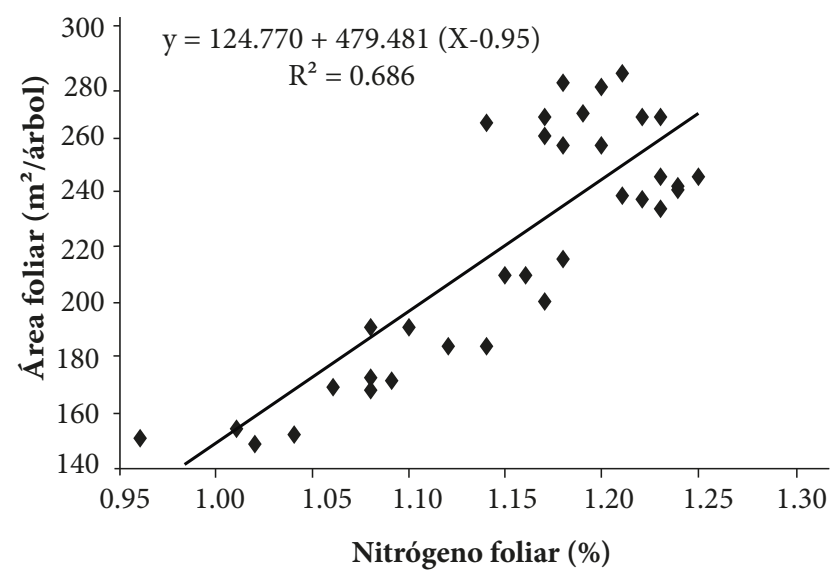

Figura 5. Regresión entre el área foliar de árboles de litchi var. 'Brewster' y la concentración de $\mathrm{N}$ foliar, en Tuxtepec, Oaxaca.

de nutrimentos o creciendo en un suelo con mayor nivel de fertilidad.

\section{CONCLUSIONES}

Se concluye que los árboles de litchi en huertos del norte del Estado de Oaxaca están en condiciones deficientes de $\mathrm{N}, \mathrm{P}$ y K por la falta de abastecimiento de nutrimentos, y que la fertilización con NPK y micronutrimentos eleva la floración, el rendimiento de fruto, el área foliar y la producción anual de biomasa, el contenido foliar de clorofila, y las concentraciones foliares de N, P y K. También se concluye que al aumentar el contenido de $\mathrm{N}$ foliar hay incrementos en el peso de brotes y en área foliar.

\section{AGRADECIMIENTOS}

A la Fundación Produce Oaxaca, a la LPI-8 del Colegio de Postgraduados y al CONACYT, por el apoyo financiero.

\section{BIBLIOGRAFÍA}

Abadía J J (1997) Methods of diagnosis of mineral nutrition of plants, a critical review. Mineral nutrition and fertilizers use for deciduous fruit crops. Acta Hort. 448:179-192.

Aserca, agregar nombre completo (2004) Apoyos y servicios a la comercialización agropecuaria. Rev. Claridades Agrop. 74:32-48.

Barrantes O, C A Gracia (1989) Estimación del área foliar a partir de la superficie de Albura en Pinus sylvestris. Options Medit. 3:5356.

Batten D J, C A McConchie (1995) Floral induction in growing buds of lychee (Litchi chinensis) and mango (Mangifera indica). Aust. J. Plant Physiol. 22:783-791.

Bremmer J M (1965) Total nitrogen. In: Methods of Soil Analysis. C A Black (ed). Part 2. American Society of Agronomy. Madison Wisconsin. pp:1149-1178.

Caballero A, L Martínez, J Bernárdez (1993) Tablas Matemáticas. Ed. Esfinge. Edo. México. 14 p.

Castellanos R J Z, J L Reyes C (1982) La Utilización del Estiércol en la Agricultura. Ingenieros Agrónomos del Tecnológico de Monterrey, A.C. Torreón, Coah. México. 154 p.

Chapman H, P Pratt (1979) Métodos de Análisis para Suelos, Plantas y Aguas. Ed. Trillas. México, D.F. 195 p.

Cochran W G, G M Cox (1965) Diseños Experimentales. Ed. Trilas. México, D.F. 618 p.

Cronje R, D Sivakumar, P Mostert, L Korsten (2009) Effect of different preharvest treatment regimens on fruit quality of Litchi cultivar “Mauritius". J. Plant Nutr. 32:19-29.

Fan X L, C L Huang, U Juhani, D Danny (2005) NPK nutrition dynamics of lychee during the annual growth cycles. Acta Hort. 665 p.

Garcés F, C A Forcelini (2011) Peso de hojas como herramienta para estimar el área foliar en soya. Cien. Tecnol. 4:13-18.

Hieke S, Menzel C M, P Lüdders (2002) Effects of leaf, short and fruit development on photosynthesis of lychee trees (Litchi chinensis). Tree Physiol. 22:955-961.

Hundal H S, C L Arora (1993) Nutrient status of litchi (Litchi chinensis Sonn) orchards in submontaneous area of Punjab. Indian Soc. Soil Sci. 41:778-779.

Kozlowski T, P Kramer, S Pallardy (1991) The Physiological Ecology of Woody Plants. Academic Press. New York. 657 p.

Kumar R (2010) Evaluation of dynamic substrates under intergrated plant nutrient management affecting growth yield and quality of $\mathrm{Li}$ tchi (Litchi chinensis Sonn.) Acta Hort. 863:443-448.

Li L -J, D -H Zeng, Z -Y Yu, Z -P Fan, R P Mao, P L Peri (2011) Foliar N/P ratio and nutrient limitation to vegetation growth on Keerqin sandy grassland of North-east China. J. British Grassland 66:237-242.

Lynch S, S Golweber, C Rich (1974) Some effects of nitrogen, phosphorus and potassium on the yield, tree growth and leaf analysis of avocado. Proc. Florida Sta. Hort. Soc. 67:220-223.

Menzel C M (1983) The control of floral initiation in Lychee: A review. Sci. Hort. 21:201-215.

Menzel C M (1984) The pattern and control of reproductive development in lychee: a review. Sci. Hort. 22:333-45.

Menzel C M, D R Simpson (1987) Lychee nutrition: a review. Sci. Hort. 31:195-224

Menzel C M, M L Carseldine, G F Haydon, D R Simpson (1992) A review of existing and proposed new leaf standards for lychee. Sci. Hort. 40:33-53.

Menzel C M (2002) Lychee production in Australia. In: Lychee Production in the Asia-Pacific Region. M K Papademetriou, FJ Dent (eds) Food and Agricultural Organization of the United Nations Regional Office for Asia and the Pacific. Bangkok, Thailand. pp:14-27.

Menzel C M, D R Simpson (1994) Lychee. In: Handbook of Environmental Physiology of Fruit Crops. B Schaffer, P C Andersen (eds) CRC Press 2:123-145 
Menzel C M, T S Rasmussen, D R Simpson (1995) Carbohydrate reserves in lychee trees (Litchi chinensis Sonn.). J. Hort. Sci. 70:245-255.

Menzel C M, G K Waite (2005) Litchi and longan botany, production and uses. CABI Publishing. 297 p.

Mitra S (2002) Overview of lychee production in the Asia Pacific Region. In: Lychee Production in the Asia-Pacific Region. M K Papademetriou, F J Dent (eds). Food and Agricultural Organization of the United Nations, Regional Office for Asia and the Pacific. Bangkok, Thailand. pp:5-13.

Pathak K P, S K Mitra (2010) Rate and time of potassium fertilization influence yield and quality of Litchi. Acta Hort. 863:235-242.

Pratt P F, F E Broadbent, J P Martin (1973) Using organic wastes as nitrogen fertilizers. California Agric. 27:10-13.

Ray R, P Singh (1989) Leaf area estimation in Capsicum (Capsicum annuum L.). Sci. Hort. 39:181-188.
SAS Institute (2003) SAS/STAT ${ }^{\circledR}$ User's Guide. Version 9.0 SAS Institute Inc., Cary, NC: pp:209-243.

SIAP, Servicio de Información y Estadística Agroalimentaria y Pesquera (2010) Servicio de Información y Estadística Agroalimentaria y Pesquera. SAGARPA. México. Disponible en: http://www. siap.sagarpa.org.mx (Septiembre 2011).

Singh H P, S Babita (2002) Lychee production in india. In: Lychee Production in the Asia-Pacific Region. M K Papademetriou, FJ Dent (eds). Food and Agricultural Organization of the United Nations, Regional Office for Asia and the Pacific. Bangkok, Thailand. pp:55-77.

Soil Survey Staff (1994) Keys to Soil Taxonomy. Soil Conservation Service. U.S. Departament of Agriculture. PO Box 2890. Washington, D.C. 20013.

Valdivia J S, A Trinidad-Santos, R A Muñoz-Márquez T (2010) Nutritional survey of lychee on the Coast area of the Gulf of México. Acta Hort. 863:413-423. 